\title{
The cyclin-dependent kinase inhibitor flavopiridol sensitizes human hepatocellular carcinoma cells to TRAIL-induced apoptosis
}

\author{
KAZUMI MIYASHITA, KATSUYA SHIRAKI, HIROYUKI FUKE, TOMOKO INOUE, YUTAKA YAMANAKA, \\ YUMI YAMAGUCHI, NORIHIKO YAMAMOTO, KEIICHI ITO, KAZUSHI SUGIMOTO and TAKESHI NAKANO
}

First Department of Internal Medicine, Mie University School of Medicine, Tsu, Mie, Japan

Received March 1, 2006; Accepted May 2, 2006

\begin{abstract}
Flavopiridol was one of the first cyclin-dependent kinase inhibitors demonstrated to have an antitumor effect in several cancer types. Here, we investigated the effects of flavopiridol on TNF-related apoptosis-inducing ligand (TRAIL) in the human hepatocellular carcinoma (HCC) cell lines HLE and HepG2, and evaluated the role of flavopiridol in apoptosis. To better understand the mechanism of increased TRAIL sensitivity in HCC cells, we determined the effect of flavopiridol on cell surface expression of TRAIL and TRAIL receptors using flow cytometry analysis. The levels of survivin, FLIP, Bcl-xL and X-chromosome-linked IAP (XIAP) in treated and untreated cells was also determined. Flavopiridol decreased cell viability in a dose-dependent manner in the two HCC cell lines tested. The pan-caspase inhibitor z-VAD-FMK did not inhibit the effect. However, subtoxic levels of flavopiridol dramatically enhanced TRAIL-induced apoptosis in both cells. Flavopiridol up-regulated TRAIL, TRAIL-R1 and TRAIL-R2 in both cell lines. In addition, flavopiridol down-regulated expression of survivin in both cell lines, and expression of FLIP and Bcl-xL were down-regulated in HLE cells. In summary, flavopiridol augmented TRAIL sensitivity by up-regulation of TRAIL receptors and down-regulation of survivin, FLIP and Bcl-xL. Thus, combining flavopiridol with a TRAIL agonist may prove to be an effective new strategy for treatment of HCC.
\end{abstract}

\section{Introduction}

Human hepatocellular carcinoma (HCC) is a rapidly progressive tumor. In HCC, the balance between cell proliferation and cell death may play a particularly important role (1).

Correspondence to: Dr Katsuya Shiraki, First Department of Internal Medicine, Mie University School of Medicine, 2-174 Edobashi, Tsu, Mie 514-8507, Japan

E-mail: Katsuyas@clin.medic.mie-u.ac.jp

Key words: cyclin-dependent kinase inhibitor, flavopiridol, hepatocellular carcinoma, TNF-related apoptosis-inducing ligand, survivin, FLIP, Bcl-xL
Hepatocytes may be particularly prone to damage and transformation since they are a regenerative cell type with a rapid proliferative rate. Thus, a failure to appropriately induce apoptosis may be particularly important to the development of HCC.

Perturbation of cell cycle regulation is important to cancer, including HCC. Recent studies indicated that inappropriate expression of the cell cycle related proteins cyclin D1, cyclin dependent kinase 4 (Cdk4), and cyclin $\mathrm{E}$ is one of the major factors that contributes to HCC development $(2,3)$. In HCC, cyclin D1-related kinase activity is accompanied by upregulation of $\mathrm{Cdk} 4$ activity but not Cdk6 activity. In addition, the protein levels and kinase activities of cyclin D1, Cdk4, and cyclin $\mathrm{E}$ were higher in poorly differentiated HCC and advanced HCC than in normal cells. Long-term overexpression of cyclin D1, Cdk4, cyclin E and cyclin A was detected not only in preneoplastic cells but also in neoplastic cells of the liver. These observations indicate that cyclins and $\mathrm{Cdks}$ may be critical factors in the development of HCC, with contributions to HCC progression and histopathologic grade $(2,3)$. On the other hand, most HCC cells show strong resistance to various stimuli that otherwise induce apoptosis in proliferating cells. In HCC cells, several cellular anti-apoptotic mechanisms, including reduced expression of Fas or caspase and expression of anti-apoptotic proteins such as FLICE-inhibitory protein (FLIP), the Bcl-2 family, heat shock protein, and inhibitors of apoptosis (IAP) families, or receptor-mediated survival signals, contribute to $\mathrm{HCC}$ resistance against immunologic cytotoxity (4).

This evidence suggests that drugs that regulate both the cell cycle and apoptosis will be needed to establish effective therapies for HCC. Flavopiridol is a semi-synthetic flavonoid and the first $\mathrm{Cdk}$ inhibitor to enter clinical trials (5-7). It strongly inhibits Cdk1, Cdk2, Cdk4 and Cdk7 and causes cytostatic or cytotoxic effects in various human cancer cell lines (8-9). By inhibiting Cdks, flavopiridol causes arrest of the cell cycle in G1-S and G2-M boundary phases (8,10-14). Flavopiridol has also been shown to induce apoptosis in noncycling transformed cells in a p53-independent manner and to down-regulate several proteins, including cyclins D and B, vascular endothelial growth factor, X-linked IAP (XIAP), cIAP-2, Mcl-1, Bcl-2, survivin and p21 in breast, colon, lung, gastric and prostate cancer and in leukemia cells $(8,10,15-17)$. 
In a recent study, expression of the TNF family proteins TNF- $\alpha$ and TNF-related apoptosis-inducing ligand (TRAIL) was shown to correlate with lethality of flavopiridol in some cell lines $(10,15)$. TRAIL selectively induces apoptosis in various transformed cell lines but not more normal tissues (18). TRAIL can induce apoptosis by interaction with two receptors, referred to as TRAIL-R1 and -R2 (or DR4 and DR5, respectively, for death receptor 4 and 5). Each of these receptors has a death domain that mediates cellular apoptosis. TRAIL-R1 and -R2 are expressed in malignant cells as well as in normal tissues. We previously reported that treatment with the conventional chemotherapeutic agents doxorubicin and camptothecin dramatically augmented TRAIL-induced cytotoxity in most HCC cells (18). However, the anti-HCC mechanisms of flavopiridol and the effect of the combination of flavopiridol and TRAIL on HCC cells have not been completely elucidated. Therefore, we investigated the effects of flavopiridol on human HCC cells and evaluated the cytotoxic mechanism, especially as it relates to the apoptotic pathway.

\section{Materials and methods}

Materials. Flavopiridol was a kind gift from Aventis Pharmaceuticals (Bridgewater, NJ). A stock solution of flavopiridol was made at a concentration of $10 \mathrm{mM}$ in DMSO and stored at $-20^{\circ} \mathrm{C}$.

Cell lines. The HCC cell line HLE was purchased from the Health Science Research Resources Bank (Osaka, Japan). The human HCC cell line HepG2 was purchased from the American Type Culture Collection. HCC cell lines were cultured in Dulbecco's modified Eagle's medium at $37^{\circ} \mathrm{C}$. All media were supplemented with $1 \%$ penicillin/streptomycin (Invitrogen, Carlsbad, CA) and 10\% heat-inactivated fetal calf serum (Invitrogen).

Detection of apoptosis-related proteins by immunoblotting. Expression of survivin, FLIP, XIAP and Bcl-xL in HCC cell lines were analyzed by immunoblotting. Briefly, cells were harvested after stimulation with flavopiridol (0-500 nM). Cells were then lysed on ice in lysis buffer $(50 \mathrm{mM} / 1$ Tris- $\mathrm{HCl}$ $\mathrm{pH} 8.0,150 \mathrm{mM} / 1 \mathrm{NaCl}, 5 \mathrm{mM} / 1$ ethylenediaminetetraacetic acid, 1\% NP40, $1 \mathrm{mM}$ phenylmethylsulfonyl fluoride). After centrifugation, supernatants were collected and protein content was measured using the Bio-Rad protein assay kit (Bio-Rad Laboratories, Hercules, CA). Equal amounts of protein from each extract were separated by $14 \%$ sodium dodecyl sulfatepolyacrylamide gel electrophoresis (SDS-PAGE) and transferred onto nitrocellulose membranes (Toyo Roshi, Tokyo, Japan) using the Bio-Rad electrotransfer system (Bio-Rad Laboratories). Blots were blocked by incubation in 5\% milk with Tris- $\mathrm{HCl} \mathrm{pH} 7.5$ and $0.1 \%$ Tween-20 for $2 \mathrm{~h}$ at room temperature and probed overnight at $4^{\circ} \mathrm{C}$ with mouse antisurvivin monoclonal antibody (Santa Cruz Biotechnology, Santa Cruz, CA), mouse anti-FLIP monoclonal antibody (MBL, Nagoya, Japan), rabbit anti-Bcl-xL polyclonal antibody (Transduction; Lexington, KY) or mouse anti-XIAP monoclonal antibody (Transduction). Antibodies were diluted 1:1000 with $5 \%$ milk in Tris- $\mathrm{HCl}(\mathrm{pH} 7.5)$ and $0.1 \%$ Tween-20. The

\section{(a) HLE}

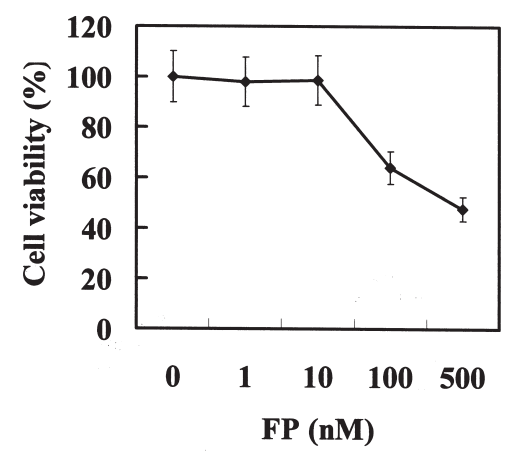

(b) HepG2

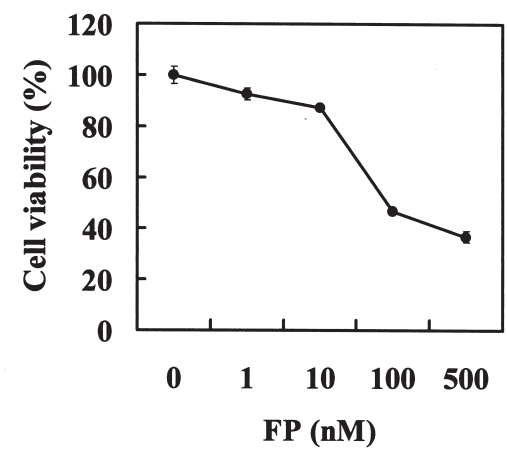

Figure 1. Effect of flavopiridol on HCC cell lines. (a) HLE, (b) HepG2. HCC cells were incubated with various concentrations of flavopiridol for $24 \mathrm{~h}$. Cell viability was assessed using the MTT assay. The data shown are the mean \pm SD of six independent experiments.

immunoblots were then probed with horseradish peroxidaseconjugated anti-mouse IgG or horseradish peroxidaseconjugated anti-rabbit IgG (diluted 1:2000 with 5\% milk in Tris- $\mathrm{HCl} \mathrm{pH} 7.5)$. After the final wash, the signal was detected with an ECL kit (Amersham Pharmacia Biotech, Buckinghamshire, UK).

Detection of apoptosis. A total of $5 \times 10^{4}$ HLE or HepG2 cells were cultured in $35-\mathrm{mm}$ culture dishes for $24 \mathrm{~h}$, followed by the addition of $10 \mathrm{ng} / \mathrm{ml}$ recombinant human TRAIL (R\&D Systems, Minneapolis, MN) and/or $100 \mathrm{nM}$ flavopiridol. After incubation for $24 \mathrm{~h}$, cell nuclei were stained with 4'6,diamidino-2-phenylindole (DAPI; Sigma, St Louis, MO) and observed under a fluorescence microscope (Zeiss, Göttingen, Germany). To assess the viability of HCC cells, the 3-(4,5dimethylthiazol-2-yl)-2,5-diphenyl tetrazolium bromide (MTT) assay was performed. The HCC cells were plated at a density of $1.0 \times 10^{4}$ cells/well in 96-well flat-bottom microtiter plates (Corning Glass Works, Corning, NY) and each plate was incubated for $24 \mathrm{~h}$ at $37^{\circ} \mathrm{C}$ in $5 \% \mathrm{CO}_{2}$. Next, flavopiridol 0 $500 \mathrm{nM}$ was added in the absence or presence of $0-100 \mathrm{ng} / \mathrm{ml}$ TRAIL (R\&D Systems), 0-100 ng/ml TNF- $\alpha$ (Cosmo Bio Co., Tokyo, Japan), or 0-100 ng/ml anti-Fas agonistic antibody (MBL), and the plates ware incubated for $24 \mathrm{~h}$. Then, the live cell count was assayed with a Cell Titer 96 assay kit (Promega, Madison, Wisconsin) according to the manufacturer's instructions. The absorbance of each well was measured with a microtiter plate reader (Bio-Rad) at $570 \mathrm{nM}$. 
(a) HLE

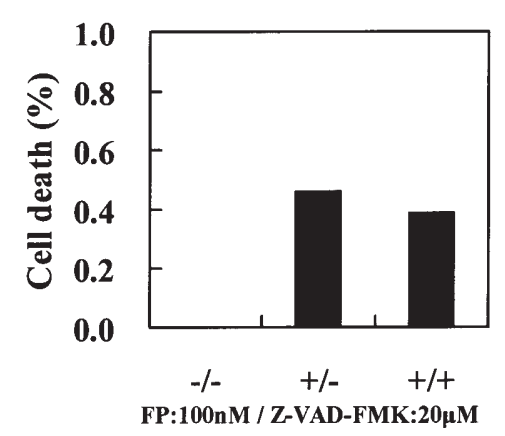

(b)HepG2

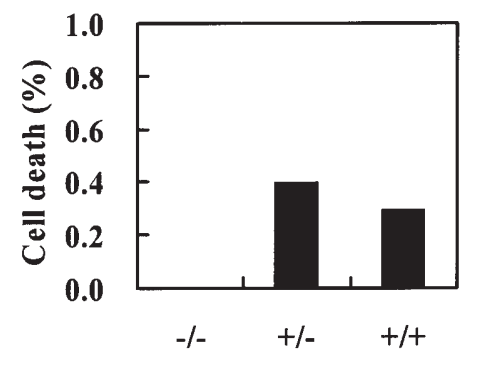

FP:100nM / Z-VAD-FMK:20 $\mu$ M

Figure 2. Influence of z-VAD-FMK on the changes in viability of HCC cells induced by flavopilidol. (a) HLE, (b) HepG2. HCC cells were incubated in the absence or presence of $100 \mathrm{nM}$ flavopiridol for $24 \mathrm{~h}$. Cell viability was assessed using the MTT assay. The data shown are the mean \pm SD of six independent experiments.

Flow cytometric analysis. Approximately $5 \times 10^{6}$ cells of each cell line cultured with or without $100 \mathrm{nM}$ flavopiridol for $48 \mathrm{~h}$ were washed twice with $1 \%$ bovine serum albumin in phosphate-buffered-saline (PBS) and incubated for $2 \mathrm{~h}$ on ice with $5 \mu \mathrm{g} / \mathrm{ml}$ PE anti-human TRAIL, PE-conjugated antihuman TRAIL-R1, PE-conjugated anti-human TRAIL-R2 or PE-conjugated mouse IgG3 isotype control (eBioscience, San Diego, CA ). After two washes with PBS the cells were analyzed for TRAIL, TRAIL-1 and TRAIL-2 expression by FACScan using CellQuest software (Becton Dickinson, Tokyo, Japan).

zVAD inhibition. An MTT assay was performed to assess the influence of flavopiridol after treatment with z-VAD-FMK, a general caspase inhibitor. The HCC cells were plated at a density of $1 \times 10^{4}$ cells/well in 96-well flat-bottom microtiter plates and each plate was incubated for $24 \mathrm{~h}$ at $37^{\circ} \mathrm{C}$ in $5 \%$ $\mathrm{CO}_{2}$. We treated cells with $100 \mathrm{nM}$ flavopiridol, followed by $20 \mu \mathrm{M} \mathrm{z}-\mathrm{VAD}-\mathrm{FMK}$ and the plates were incubated for $24 \mathrm{~h}$. Finally, the live cell count was assayed using a Cell Titer 96 assay kit (Promega, Madison, Wisconsin) according to the manufacturer's instructions. The absorbance of each well was measured on a microtiter plate reader (Bio-Rad) at $570 \mathrm{nM}$.

\section{Results}

Flavopiridol reduced viability of $\mathrm{HCC}$ cells. To investigate change in viability in response to flavopiridol, HCC cells (a) HLE

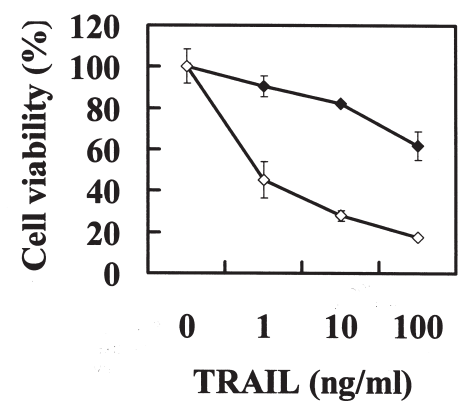

\section{(b)HepG2}

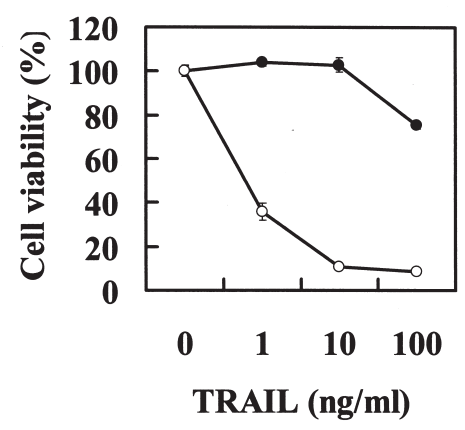

Figure 3. Influence of flavopiridol on the TRAIL-induced apoptosis pathway in HCC cell lines. (a) HLE, (b) HepG2. HCC cells were incubated for $24 \mathrm{~h}$ with various concentrations of TRAIL in the absence $(\bullet)$ or presence of a subtoxic level of flavopiridol $(100 \mathrm{nM} ; \circ)$. Cell viability decreased in response to TRAIL in a dose-dependent manner. In combination, flavopiridol and TRAIL caused a synergistic reduction in cell viability in both cell lines tested. Cell viability was assessed using the MTT assay. The data shown are the mean \pm SD of six independent experiments.

were incubated with various concentrations of flavopiridol for $24 \mathrm{~h}$. Cell viability was assessed by the MTT assay. Flavopiridol decreased cell viability in the HCC cell lines HLE and HepG2 in a concentration-dependent manner (Fig. 1). When these cells were incubated with $500 \mathrm{nM}$ of flavopiridol, the percentage of viable cells was $48 \%$ for HLE and $40 \%$ for HepG2 cells.

In order to ask if flavopiridol can induce apoptosis in HCC cells, we next assessed DAPI staining $24 \mathrm{~h}$ after treatment with $100 \mathrm{nM}$ flavopiridol . Flavopiridol-treated HLE cells did not show features typical of apoptosis (Fig. 6b); the same result was observed for HepG2 cells (data not shown). Despite this, reduced viability in these cell lines prompted us to analyze apoptosis-related factors in these cells more rigorously. Thus, we next examined the effect of a caspase inhibitor on flavopiridol-treated cells. In order to determine whether or not the caspase pathway is related to the apoptosis of HCC cells induced by flavopiridol, we examined the changes in viability of HCC cells treated with flavopiridol and with or without z-VAD-FMK, a strong pan-caspase inhibitor. However, z-VAD-FMK did not have a detectable effect on cell viability in either cell line (Fig. 2). Collectively, the results suggest that the reduction of cell viability observed for flavopiridol is independent of the apoptosis pathway.

Flavopiridol augmented TRAIL, TNF- $\alpha$ and anti-Fas agonistic antibody-induced apoptosis. It is well known that TRAIL 
(a) HLE

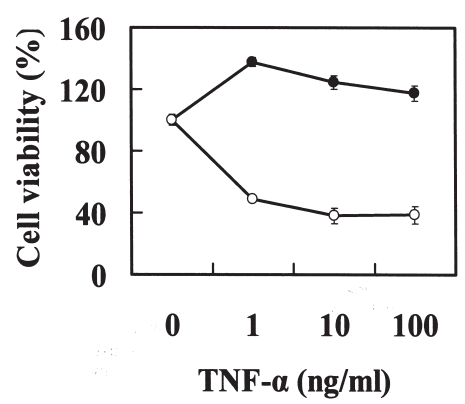

(b) HepG2

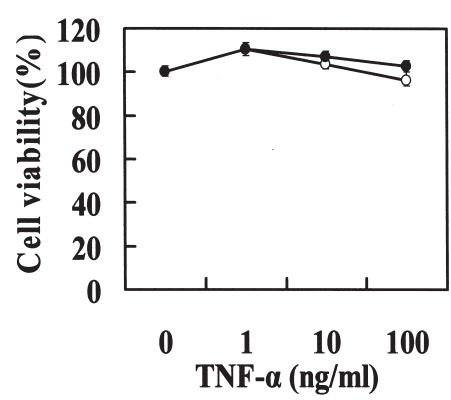

Figure 4. Influence of flavopiridol on the TNF- $\alpha$-induced apoptosis pathway in HCC cell lines. (a) HLE, (b) HepG2. HCC cells were incubated for $24 \mathrm{~h}$ with various concentrations of TNF- $\alpha$ in the absence $(\bullet)$ or presence of a subtoxic level of flavopiridol $(100 \mathrm{nM} ; 0)$. TNF- $\alpha$ and flavopiridol caused a synergistic reduction in cell viability in HLE cells but not in HepG2 cells. Cell viability was assessed using the MTT assay. The data shown are the mean $\pm \mathrm{SD}$ of six independent experiments.

induces apoptosis in HCC cells via a caspase cascade. To examine the relationship between flavopiridol and TRAILinduced apoptosis, HCC cells were incubated with various concentrations of TRAIL in the presence or absence of subtoxic levels of flavopiridol $(100 \mathrm{nM})$ for $24 \mathrm{~h}$. Cell viability decreased in response to TRAIL in a dose-dependent manner in both cell lines (Fig. 3). Moreover, treatment with both TRAIL and flavopiridol had a synergistic effect on reduction of cell viability (Fig. 3).

To examine whether, when combined, TRAIL and flavopiridol induce apoptosis in HCC cells, we next assessed DAPI staining $24 \mathrm{~h}$ after treatment with TRAIL $(10 \mathrm{ng} / \mathrm{ml})$ and flavopiridol $(100 \mathrm{nM})$. We did not observe induction of apoptosis in TRAIL-treated HLE cells (Fig. 6a) or in Hepg2 cells (data not shown). However, cells treated with both TRAIL and flavopiridol showed features typical of apoptosis, including nuclear condensation and nuclear fragmentation (Fig. 6c and d). These results indicate that flavopiridol may affect the TRAIL-induced apoptosis pathway in HCC cells.

Next, we examined the effect of flavopiridol on TNF- $\alpha$ and anti-Fas agonistic antibody-induced apoptosis. Flavopiridol synergistically augmented TNF- $\alpha$-induced apoptosis in HLE cells (Fig. 4a) but not in HepG2 cells (Fig. 4b). In addition, flavopiridol enhanced anti-Fas agonistic antibody-induced apoptosis in both cell types (Fig. 5a and b).

Flavopiridol up-regulated expression of TRAIL, TRAIL-RI and $T R A I L-R 2$. In order to examine the mechanisms of increased (a) HLE

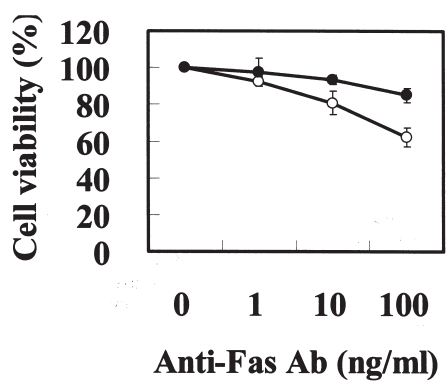

(b) HepG2

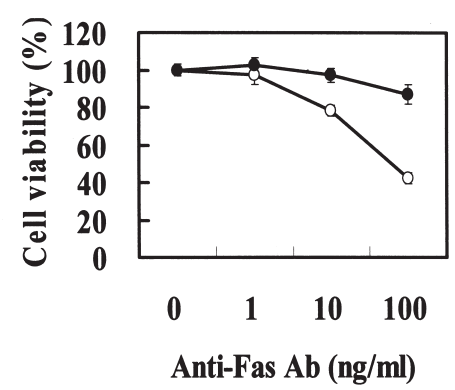

Figure 5. Influence of flavopiridol on anti-Fas agonistic antibody-induced apoptosis in HCC cell lines. (a) HLE, (b) HepG2. HCC cells were incubated for $24 \mathrm{~h}$ with various concentrations of anti-Fas agonistic antibody in the absence $(\bullet)$ or presence of a subtoxic level of flavopiridol $(100 \mathrm{nM} ; \circ)$. Cell viability decreased in response to anti-Fas agonistic antibody in a dosedependent manner; furthermore, anti-Fas agonistic antibody in combination with the subtoxic level of flavopiridol caused a synergistic reduction of cell viability in both cell lines. Cell viability was assessed using the MTT assay. The data shown are the mean \pm SD of six independent experiments.

TRAIL susceptibility in flavopiridol-treated HCC cells, we looked at the cell surface expression of TRAIL and TRAIL receptors in HCC cells (HLE and HepG2) using flow cytometric analysis. After $48 \mathrm{~h}$ treatment with $100 \mathrm{nM}$ flavopiridol, expression of TRAIL, TRAIL-R1 and TRAIL-R2 was significantly up-regulated in both cell types (Fig. 7).

Flavopiridol suppressed the apoptosis-related proteins survivin, FLIP and Bcl-xL. Next we investigated the effects of flavopiridol on the levels of anti-apoptotic proteins, since these proteins play a major role in controlling apoptotic pathways (19-22). To determine the relative levels of a select group of apoptosis-related proteins, we used immunoblotting to detect survivin, FLIP and XIAP, which are direct inhibitors of cell-death proteases, and Bcl-xL, which is an apoptosis inhibitory protein. In both cell types, detectable levels of survivin were reduced in response to flavopiridol treatment in a concentration-dependent manner (Fig. 8). Moreover, FLIP and $\mathrm{Bcl}-\mathrm{xL}$ levels were reduced in a concentration-dependent manner in HLE cells but not in HepG2 cells (Fig. 8). Expression of XIAP was not influenced by flavopiridol treatment in either cell line (Fig. 8).

\section{Discussion}

In this study, we analyzed the cytotoxic effects of flavopiridol on human hepatocellular carcinoma (HCC) cells. Flavopiridol 
a

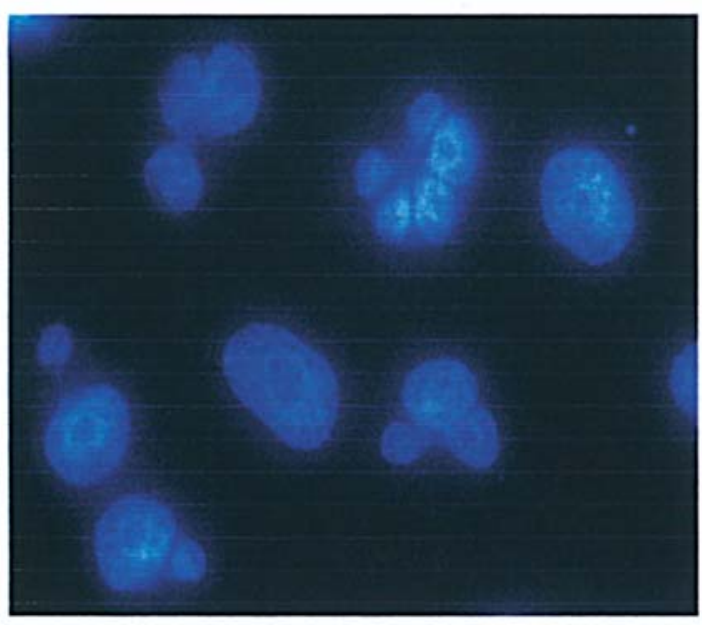

c

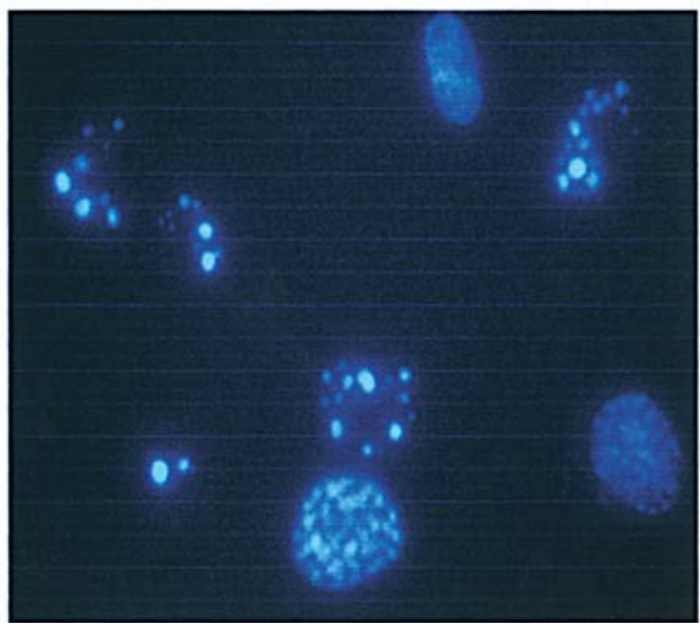

b

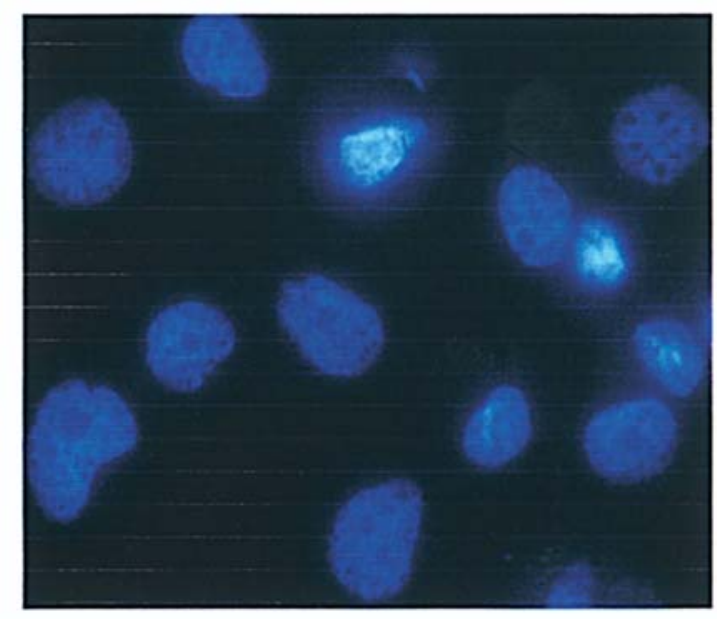

d

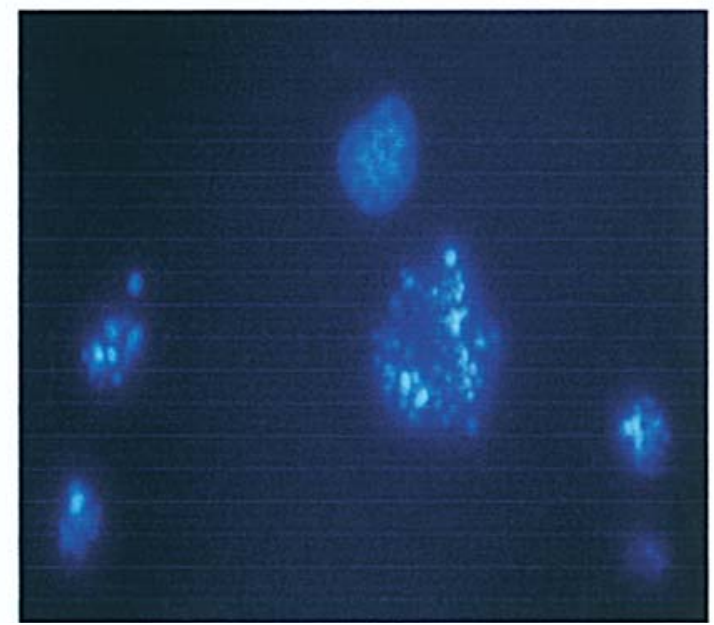

Figure 6. Influence of flavopiridol on TRAIL-induced apoptosis in HLE cells. HLE cells were incubated for $24 \mathrm{~h}$ with TRAIL (10 ng/ml) in the absence or presence of a subtoxic level of flavopiridol $(100 \mathrm{nM})$. Although apoptosis was not induced in TRAIL-treated HLE cells (a) or in flavopiridol-treated HLE cells (b), TRAIL and flavopiridol-treated HLE cells showed typical apoptotic features, including nuclear condensation and nuclear fragmentation (DAPI staining in $\mathrm{c}$ and $\mathrm{d}$ ).

decreased cell viability in HCC cells in a concentrationdependent manner. When these cells were incubated with $500 \mathrm{nM}$ of flavopiridol, viability of both HLE and HepG2 cells decreased. This effect was addressed further by asking if caspases play a role in the reduction of cell viability observed after flavopiridol treatment. We found that the change in HCC cell viability in response to flavopiridol was not suppressed by z-VAD-FMK, a strong pan-caspase inhibitor. These results suggest that the caspase-mediated pathway is not involved in flavopiridol-mediated cytotoxicity.

Flavopiridol is a semi-synthetic flavonoid and the first cyclin-dependent kinase ( $\mathrm{Cdk}$ ) inhibitor to enter clinical trials. It strongly inhibits $\mathrm{Cdk} 1, \mathrm{Cdk} 2, \mathrm{Cdk} 4$ and $\mathrm{Cdk} 7$ and causes cytostatic or cytotoxic effect on various human cancer cell lines $(8,10)$. It also broadly suppresses the transcription of genes, including cyclin D1, and binds to DNA. By inhibiting Cdks, flavopiridol arrests the cell cycle in G1-S and G2-M boundary phase $(14,23)$. In a large proportion of solid tumor cell lines, concentrations of 100-300 nM flavopiridol cause arrest at both the G1 and G2 phase of the cell cycle, consistent with inhibition of Cdks 2, 4, 6, and 1. Flavopiridol induces apoptosis in a p53-independent manner, cell death usually follows cell cycle arrest and is delayed, maximally occurring at $72 \mathrm{~h}$ after initiation of treatment and requiring concentrations higher than those required to inhibit Cdk activity (24). A recent study revealed that flavopiridol causes cells to accumulate in the G1 phase of the cell cycle $(8,25)$. In addition, the study showed that flavopiridol induces apoptosis in a manner that is associated with down-regulation of cyclins D1 and B1, as well as inhibition of Cdk1, Cdk2 and Cdk4 (8). Moreover, the effect is accompanied by a conformational change and mitochondrial localization of Bax in MB-468 cells, which are a human breast cancer-derived cell type (8). Treatment with flavopiridol also attenuated the mRNA and protein levels of the anti-apoptotic proteins XIAP, cIAP-2, Mcl-1, Bcl-xL, and survivin in some cancer cell lines $(8,13,15)$. Despite these 


\section{(a) HLE}

TRAIL
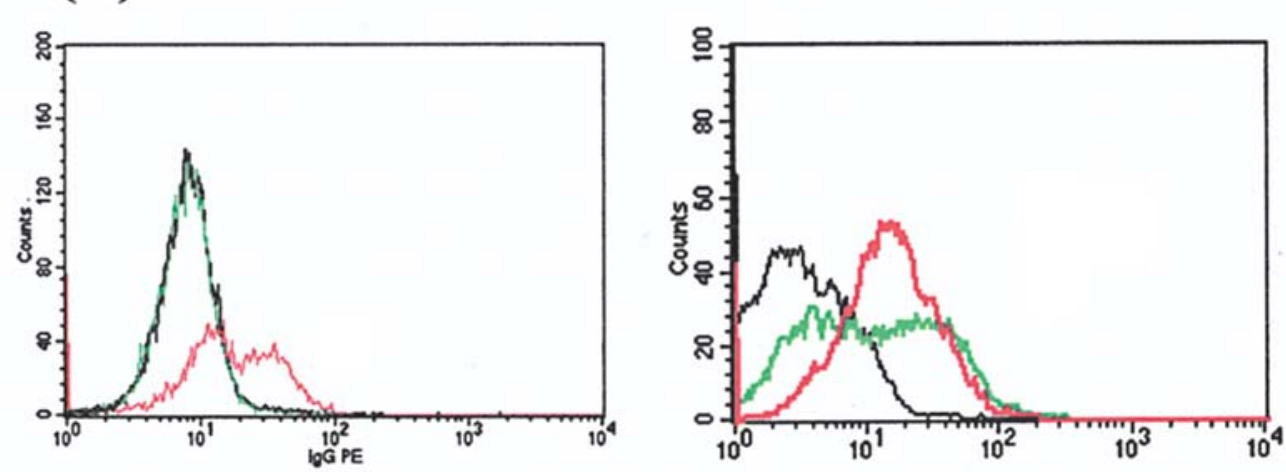
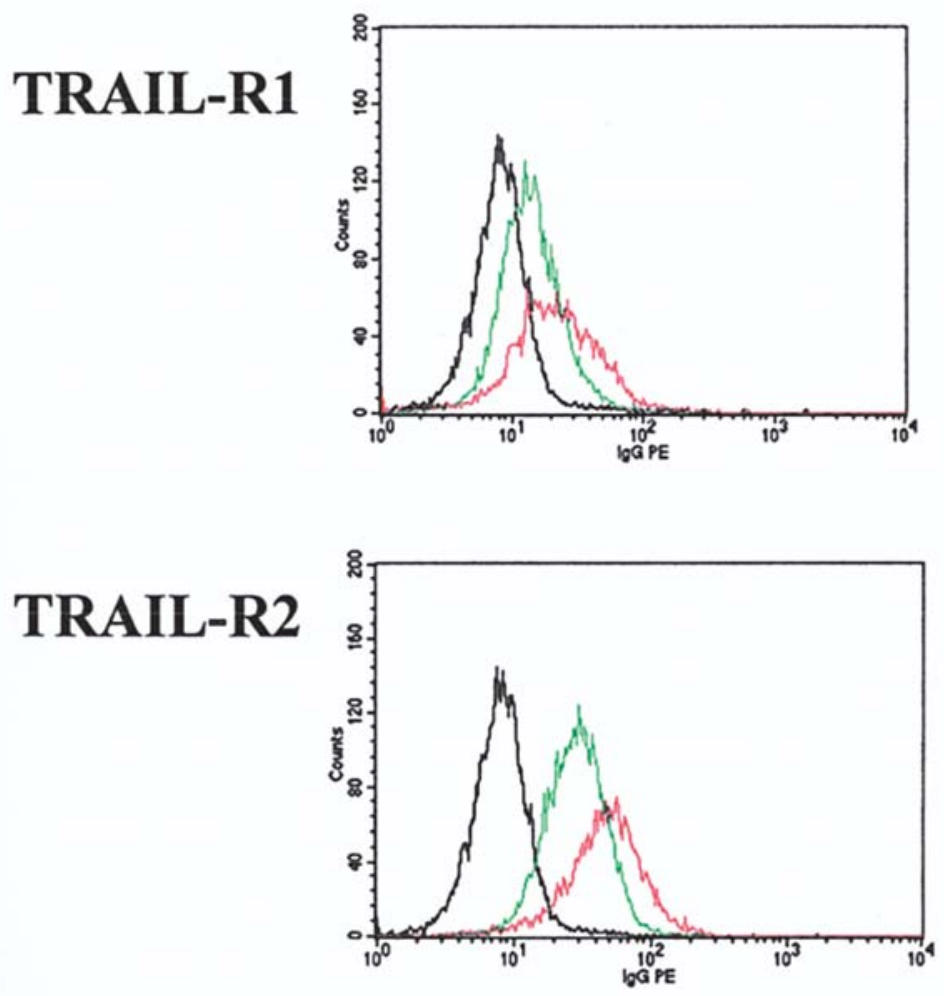

Figure 7. Influence of flavopiridol on TRAIL and TRAIL receptor expression in HCC cells. (a) HLE, (b) HepG2. HCC cells were incubated for $48 \mathrm{~h}$ in the absence or presence of flavopiridol (100 nM). HCC cells were stained using PE-anti-human TRAIL, TRAIL-R1 or TRAIL-R2 (black line, control; green line, no flavopiridol; red line, flavopiridol). Autofluorescence was determined using cells incubated with PE-control mouse IgG.

extensive studies on flavopiridol functions, the exact mechanism of the anticancer activity associated with flavopiridol remains poorly understood.

In the current study, flavopiridol augmented TRAIL, TNF- $\alpha$ and anti-Fas antibody-induced apoptosis synergistically $(10,15,25)$. These results indicate that flavopiridol can modulate sensitivity to the TNF receptor signaling pathway. We have been focusing on a TRAIL-oriented strategy for cancer therapy because TRAIL selectively induces apoptosis in various transformed cell lines but not in near-normal tissues (18). TRAIL is regulated by two receptors, TRAIL-F1 and TRAILF2, which are members of the so-called death receptor family. Each of these receptors contains a death domain that mediates cellular apoptosis. Two additional death receptors were
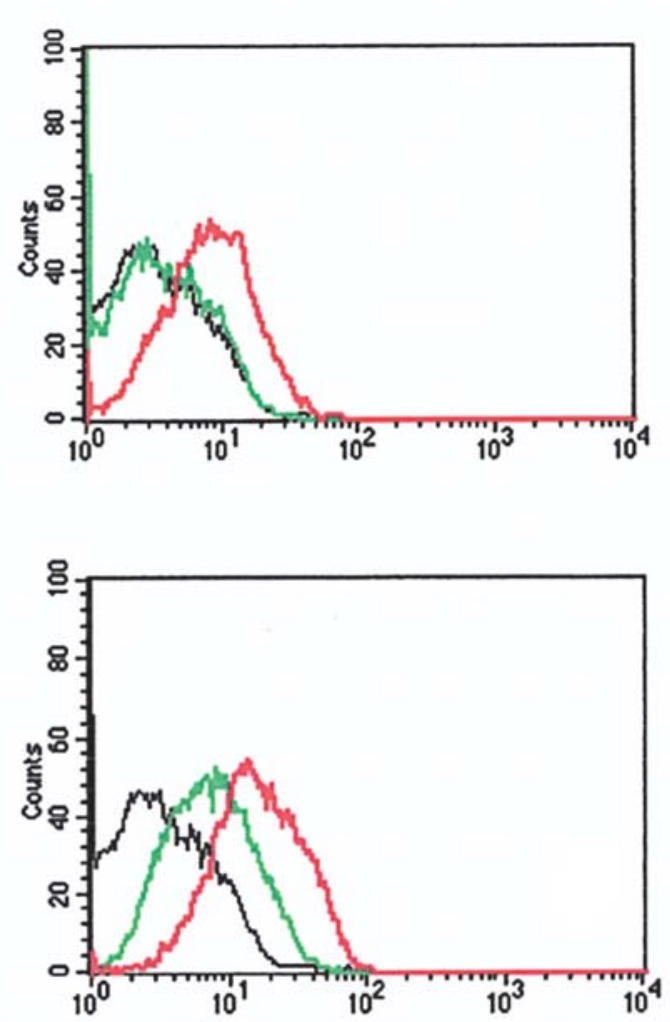

\section{(b) HepG2}

identified recently, TRAIL-R3 (or DcR1) and TRAIL-R4 (or DcR2). These receptors inhibit apoptosis by acting as decoy receptors; although they show similarity to TRAIL-R1 and -R2 in other regions, TRAIL-R3 and -R4 do not contain a cytoplasmic death domain. These decoy receptors are expressed at high levels in normal tissues and their expression levels are substantially lower in malignant cells, indicating a low cytotoxic effect in normal cells (18). Therefore, TRAIL may be a promising cytotoxic cytokine for induction of apoptosis. However, most HCC cells are resistant to TRAIL and cotreatment with chemotherapeutic agents or irradiation sensitized TRAIL-resistant cells to TRAIL. We have found that treatment with sub-cytotoxic levels of flavopiridol dramatically sensitizes HCC cells to TRAIL-induced apoptosis. Sensitization of 
(a) HLE

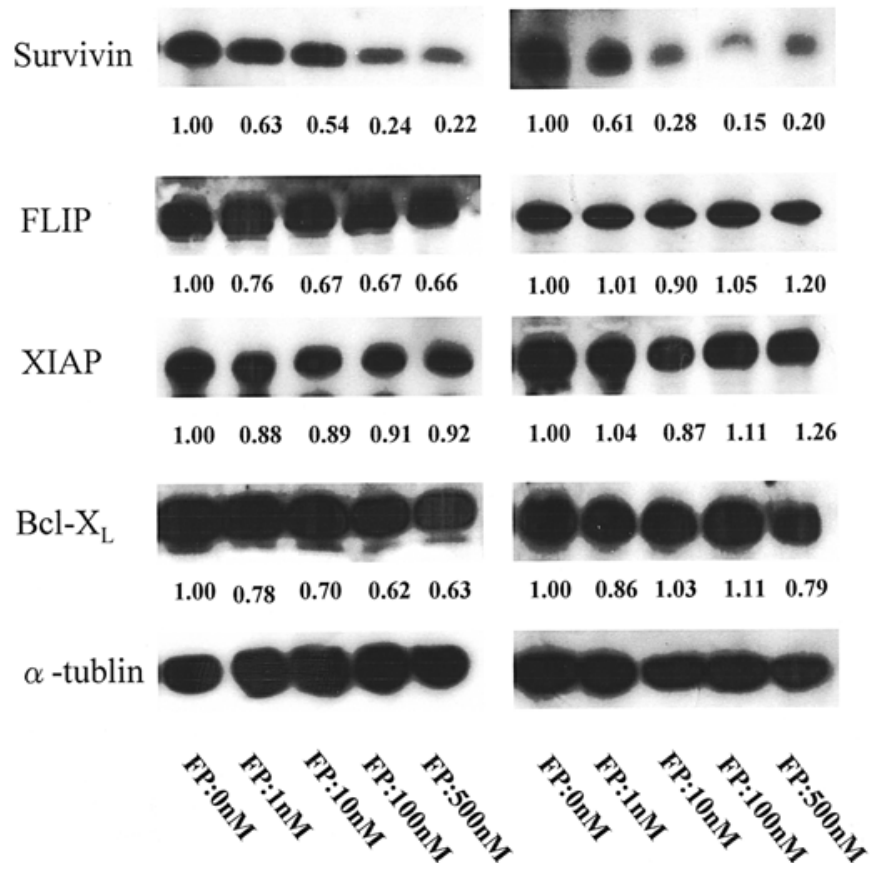

Figure 8. Changes in apoptosis-related proteins in response to flavopiridol in HCC cells. Cellular lysates ware separated by SDS-PAGE and transferred to nitrocellulose. The levels of survivin, FLIP, XIAP and Bcl-xL were detected by immunoblotting.

HCC cells to TRAIL-induced apoptosis was also independent of p53 status. p53 wild-type (HepG2) and p53 mutant-type (HLE) were similarly sensitized to TRAIL by flavopiridol, through TRAIL-R2 is transcriptionally induced by $\mathrm{p} 53$, which is activated by many chemotherapeutic agents (18).

These results indicate that flavopiridol modulates the apoptotic pathway in a way that increases sensitivity of cells to TRAIL receptor-mediated signaling. In order to better understand the mechanism of this effect, we paid special attention to inhibitors of the TRAIL receptor-mediated apoptosis pathway. First, we looked at cell surface expression of TRAIL and its receptors in HCC cells. TRAIL, TRAIL-R1 and TRAIL-R2 expression was significantly up-regulated by flavopiridol treatment in both types of HCC cells. Activation of TRAIL-R1 and -R2 has been shown to increase TRAILinduced apoptosis both in vitro and in vivo, indicating that TRAIL-R1 and -R2 are of particular importance in interpreting the TRAIL signal. Therefore, the finding that flavopiridol increased TRAIL-R1 and TRAIL-R2 expression may provide an important clue to understanding the mechanism of enhanced TRAIL sensitivity in response to flavopiridol treatment.

We next investigated the role of IAPs in the TRAIL signaling pathway, since these proteins play an important role in determining the sensitivity of apoptosis in HCC cells $(21,26)$. We also looked at expression of apoptosis-related proteins. We found that the expression of survivin was significantly down-regulated in both cells in a concentrationdependent manner. The expression of FLIP and Bcl-xL was also down-regulated in HLE cells in a concentration-dependent manner. Previous reports indicated that flavopiridol decreases XIAP, cIAP-2, Mcl-1, Bcl-xL, and survivin on mRNA levels in human breast cancer cells $(8,17)$. The mechanism by which flavopiridol down-regulates survivin, FLIP and Bcl-xL expression is not known with certainty but very likely involves flavopiridol-mediated inhibitory effects on transcription.

Survivin is the most potent member of the IAP family and blocks the action of various caspases. Moreover, IAP expression correlates with abbreviated survival, an unfavorable prognosis, resistance to therapy and accelerated rates of recurrence $(17,20)$. We previously showed that survivin is overexpressed in human HCC (19) and that it is a principal inhibitor of apoptosis via its ability to inhibit caspase-3 and -7. Survivin has also been implicated in regulating the assembly of microtubules: mouse embryos without survivin gene function lack a mitotic spindle and spindle midzone microtubules (17). We have found that survivin promotes transition from $\mathrm{G} 1$ to $\mathrm{S}$ phase and promotes cell proliferation by interacting with $\mathrm{Cdk} 4$ and releasing p21 from Cdk4 (19). These results indicate that down-regulation of survivin protein levels by flavopiridol contribute to the augmentation of TRAIL-induced apoptosis that we observed for HCC cells.

In conclusion, we have shown that flavopiridol can augment sensitivity of cells to the action of TNF family members, and TRAIL in particular, by up-regulation of TRAIL receptors and down-regulation of survivin. These results suggest that combining flavopiridol with a TRAIL agonist may be prove to be an effective new strategy for treatment of HCC.

\section{References}

1. Kalogeraki A, Garbagnati F, Santinami M and Zoras O: DNA fragmentation and cell proliferation correlated with tumor grade in patients with hepatocellular carcinoma. Cancer 96: 301-305, 2002.

2. Masaki T, Shiratori Y, Rengifo W, Igarashi K, Yamagata M, Kurokohchi K, Uchida N, Miyauchi Y, Yoshiji H, Watanabe S, Omata $\mathrm{M}$ and Kuriyama S: Cyclins and cyclin-dependent kinases: comparative study of hepatocellular carcinoma versus cirrhosis. Hepatology 37: 534-543, 2003.

3. Masaki T, Shiratori Y, Rengifo W, Igarashi K, Matsumoto K, Nishioka M and Omata M: Hepatocellular carcinoma cell cycle: study of Long-Evans Cinnamon rats. Hepatology 32: 711-720, 2000 .

4. Shiraki K, Takase $\mathrm{K}$ and Nakano $\mathrm{T}$ : The emerging role of caspase inhibitors in gastrointestinal cancers. J Gastroenterol. 37: 323-331, 2002.

5. Shapiro GI: Preclinical and clinical development of the cyclindependent kinase inhibitor flavopiridol. Clin Cancer Res 10: 4270-4275, 2004.

6. Aklilu M, Kindler HL, Donehower RC, Mani S and Vokes EE: Phase II study of flavopiridol in patients with advanced colorectal cancer. Ann Oncol 14: 1270-1273, 2003.

7. Liu G, Gandara DR, Lara PN Jr, Raghavan D, Doroshow JH, Twardowski P, Kantoff P, Oh W, Kim K and Wilding G: A Phase II trial of flavopiridol (NSC \#649890) in patients with previously untreated metastatic androgen-independent prostate cancer. Clin Cancer Res 10: 924-928, 2004.

8. Wittmann S, Bali P, Donapaty S, Nimmanapalli R, Guo F, Yamaguchi H, Huang M, Jove R, Wang HG and Bhalla K: Flavopiridol down-regulates antiapoptotic proteins and sensitizes human breast cancer cells to epothilone B-induced apoptosis. Cancer Res 63: 93-99, 2003.

9. Schwartz GK: Development of cell cycle active drugs for the treatment of gastrointestinal cancers: a new approach to cancer therapy. J Clin Oncol 23: 4499-4508, 2005.

10. Kim DM, Koo SY, Jeon K, Kim MH, Lee J, Hong CY and Jeong S: Rapid induction of apoptosis by combination of flavopiridol and tumor necrosis factor (TNF)-alpha or TNFrelated apoptosis-inducing ligand in human cancer cell lines. Cancer Res 63: 621-626, 2003.

11. Sato S, Kajiyama Y, Sugano M, Iwanuma Y and Tsurumaru M: Flavopiridol as a radio-sensitizer for esophageal cancer cell lines. Dis Esophagus 17: 338-344, 2004. 
12. Alonso M, Tamasdan C, Miller DC and Newcomb EW: Flavopiridol induces apoptosis in glioma cell lines independent of retinoblastoma and p53 tumor suppressor pathway alterations by a caspase-independent pathway. Mol Cancer Ther 2: 139-150, 2003.

13. Jiang J, Matranga CB, Cai D, Latham VM Jr, Zhang X, Lowell AM, Martelli F and Shapiro GI: Flavopiridol-induced apoptosis during $\mathrm{S}$ phase requires E2F-1 and inhibition of cyclin A-dependent kinase activity. Cancer Res 63: 7410-7422, 2003.

14. Litz J, Carlson P, Warshamana-Greene GS, Grant S and Krystal GW: Flavopiridol potently induces small cell lung cancer apoptosis during $\mathrm{S}$ phase in a manner that involves early mitochondrial dysfunction. Clin Cancer Res 9: 4586-4594, 2003.

15. Rosato RR, Dai Y, Almenara JA, Maggio SC and Grant S: Potent antileukemic interactions between flavopiridol and TRAIL/ Apo2L involve flavopiridol-mediated XIAP downregulation. Leukemia 18: 1780-1788, 2004.

16. Jung C, Motwani M, Kortmansky J, Sirotnak FM, She Y, Gonen M, Haimovitz-Friedman A and Schwartz GK: The cyclin-dependent kinase inhibitor flavopiridol potentiates gammairradiation-induced apoptosis in colon and gastric cancer cells. Clin Cancer Res 9: 6052-6061, 2003.

17. Wall NR, O'Connor DS, Plescia J, Pommier Y and Altieri DC: Suppression of survivin phosphorylation on Thr34 by flavopiridol enhances tumor cell apoptosis. Cancer Res 63: 230-235, 2003.

18. Yamanaka T, Shiraki K, Sugimoto K, Ito T, Fujikawa K, Ito M, Takase K, Moriyama M, Nakano T and Suzuki A: Chemotherapeutic agents augment TRAIL-induced apoptosis in human hepatocellular carcinoma cell lines. Hepatology 32: 482-490, 2000 .
19. Ito T, Shiraki K, Sugimoto K, Yamanaka T, Fujikawa K, Ito M, Takase K, Moriyama M, Kawano H, Hayashida M, Nakano T and Suzuki A: Survivin promotes cell proliferation in human hepatocellular carcinoma. Hepatology 31: 1080-1085, 2000.

20. Yamaguchi Y, Shiraki K, Fuke H, Inoue T, Miyashita K, Yamanaka Y, Saitou Y, Sugimoto K and Nakano T: Targeting of X-linked inhibitor of apoptosis protein or survivin by short interfering RNAs sensitize hepatoma cells to TNF-related apoptosis-inducing ligand- and chemotherapeutic agent-induced cell death. Oncol Rep 14: 1311-1316, 2005.

21. Shiraki K, Sugimoto K, Yamanaka Y, Yamaguchi Y, Saitou Y, Ito $\mathrm{K}$, Yamamoto N, Yamanaka T, Fujikawa K, Murata $\mathrm{K}$ and Nakano T: Overexpression of X-linked inhibitor of apoptosis in human hepatocellular carcinoma. Int J Mol Med 12: 705-708, 2003.

22. Okano H, Shiraki K, Inoue H, Kawakita T, Yamanaka T, Deguchi M, Sugimoto K, Sakai T, Ohmori S, Fujikawa K, Murata K and Nakano T: Cellular FLICE/caspase-8-inhibitory protein as a principal regulator of cell death and survival in human hepatocellular carcinoma. Lab Invest 83: 1033-1043, 2003.

23. Swanton C: Cell-cycle targeted therapies. Lancet Oncol 5: 27-36, 2004.

24. Wu K, D'Amico M, Wang C, Albanese C, Pestell RG and Mani S: A study of cytotoxic synergy of UCN-01 and flavopiridol in syngeneic pair of cell lines. Invest New Drugs 23: 299-309, 2005.

25. Demidenko ZN and Blagosklonny MV: Flavopiridol induces p53 via initial inhibition of Mdm2 and p21 and, independently of p53, sensitizes apoptosis-reluctant cells to tumor necrosis factor. Cancer Res 64: 3653-3660, 2004.

26. Enokimura N, Shiraki K, Kawakita T, Saitou Y, Inoue H, Okano H, Yamamoto N, Sugimoto K, Carr BI and Nakano T: Vitamin $\mathrm{K}$ analog (compound 5) induces apoptosis in human hepatocellular carcinoma independent of the caspase pathway. Anticancer Drugs 16: 837-844, 2005. 